\title{
Molecular Dynamics Simulations of the Evolution of Residual Stresses During Rapid Solidification of Aluminium
}

\author{
Michail Papanikolaou*, Konstantinos Salonitis and Mark Jolly \\ Manufacturing Theme, Cranfield University, Cranfield, MK430AL, United Kingdom \\ *Corresponding author: Michail Papanikolaou, m.papanikolaou@cranfield.ac.uk
}

\begin{abstract}
It is well known that residual stresses are quite common in castings and they emerge due to uneven cooling conditions. Nowadays, the development of atomistic modelling techniques has allowed for the in-depth investigation of the solidification process mechanics as well as the distribution of residual stresses in the simulation domain. In this study we have performed 3-dimensional Molecular Dynamics simulations to investigate the evolution of residual stresses during homogeneous nucleation in pure Aluminium as well as their distribution over the simulation domain. A simulation box containing 1 million of Aluminium atoms placed on the sites of a Face Centred Cubic (FCC) lattice has been melted and subsequently quenched under various cooling rates. The potential energy as well as the formation of grains have been monitored during quenching stages. Moreover, the present analysis is expanded to the distribution of the grain size and the number of grains as a function of the cooling rate. Finally, the obtained results suggest that the cooling rate significantly affects the distribution as well as the final magnitude of residual stresses in the solidified structure.
\end{abstract}

Keywords: Molecular Dynamics, Residual stresses, Cooling Rate, Aluminium, Grain Coarsening, Microstructure

\section{Introduction}

Since the early 1950s researchers have shown that the mechanical properties of polycrystalline materials are dependent on their grain size; the relationship between the yield strength and the grain size has been mathematically expressed by the well-known Hall and Petch relation $[1,2]$ which states that the yield strength is inversely proportional to the average grain diameter. One of the most critical parameters affecting the average grain size is the cooling rate [3,4]; the higher the cooling rate the lower the average grain size. Besides the average grain size, the cooling rate has been proven to also affect the concentration of residual stresses in the solidified component $[5,6]$ which is the topic of this study.

The first steps in solidification involve nucleation during which small aggregates (embryos) are formed. Nucleation may commence from random locations in the liquid phase (homogeneous nucleation) or might be initiated by nucleating agents, such as a foreign phase or a surface (heterogeneous nucleation) [7]. According to the Classical Nucleation Theory (CNT) these nuclei might dissolve in the liquid phase or grow in size [8]. This depends on whether the formation of a new phase is energetically favourable and can be dictated by the critical radius $r_{c}$. An embryo with a radius $r$ lower than $r_{c}\left(r<r_{c}\right)$ will probably shrink or dissolve while embryos with $r>r_{c}$ are thermodynamically stable and will grow in size becoming nuclei/grains later on during the process.

The real time observation of nano- and microscale phenomena taking place during solidification, such as nucleation, is a very challenging task [9]. On the theoretical aspect, Classical Nucleation Theory was 
proven to be capable of providing qualitatively reasonable results with respect to the nucleation rate but often inaccurate, while the major shortcoming of CNT is the assumption that the critical nucleus size is the only criterion dictating whether embryos will evolve to nuclei or not [10]. Later on, two-step nucleation models were developed. According to these models, metastable (liquid-like) clusters with high density emerge into the liquid phase; this is considered to be the first step of nucleation. The second step is the formation of crystals inside those disordered and metastable clusters [11]. Two-step nucleation theory was verified via means Molecular Dynamics (MD) simulations [12], theoretical [13] and experimental [14] studies. In general the two-step model is considered to be more accurate and representative of the real phenomena taking place during solidification compared to CNT.

On the computational side, crystal growth has been investigated via Density Functional Theory (DFT) [15], Monte Carlo simulations [16] and phase-field models [17]. However, the most widely employed numerical method for studying nucleation in nanoscale is Molecular Dynamics simulations. The first MD simulations on nucleation were performed by Tian et al. [18, 19] who investigated the rapid solidification of silver using the quantum Sutton-Chen many-body potential [20] and a relatively small number of atoms. These investigations captured the formation of metastable Body-Centred Cubic (BCC) phase prior to the stable Face-Centred Cubic (FCC) one and were also focused on the effects of the cooling rate on the grain microstructure. However, according to [21] large scale MD simulations should be performed in order to observe maximal grain sizes. Mahata et al. [22] investigated homogeneous nucleation in pure Aluminium using 1 million atoms and were able to estimate a critical nucleation temperature in accordance to CNT predictions. However, in this study MD predicted a lower critical nucleus size compared to CNT; this was attributed to (a) the simultaneous nucleation (which might affect growth) occurring at low annealing temperature values and (b) the interface energy was assumed to be constant and not temperature dependent. Shibuta et al. [23], investigated the solidification of pure iron by performing simulations with up to 12 million atoms using GPU accelerated computing. Their atomistic simulations suggested that there are 2 competing phenomena affecting homogeneous nucleation: (a) the increase of the population of the icosahedral structures into the melt and (b) the reduction of the atoms' mobility as the temperature decreases. The superposition of the aforementioned phenomena leads to a temperature value (critical temperature) for which the nucleation rate maximises. Moreover, the authors investigated both 2-dimensional and 3-dimensional solidification and were able to calculate a Avrami exponent close to 3 for 2-dimensional and 4 for 3-dimensional growth; these values are in agreement with other studies [24]. The largest MD simulation reported was also performed by Shibuta et al. [25] and included 1 billion atoms. In this investigation a local heterogeneity model was proposed; the authors observed small satellite-like grains forming in the vicinity of previously formed ones. Another topic which has been extensively studied via means of MD simulations is the effect of the cooling rate on the evolution of the potential/kinetic energy, atom type population and final grain structure [4]. Finally, in [26, 27] the effect of the cooling rate on the microstructure of pure Aluminium was also investigated and the exothermic nature of solidification was effectively captured via means of MD simulations.

In this investigation, 3-dimensional MD simulations have been performed to study homogeneous nucleation in pure Aluminium with a special focus on the grain size distribution and the evolution and distribution of residual stresses at the simulation domain while the equivalent Von Mises stress was used for the calculation of residual stresses. Four different cooling rates ranging from 0.5 to $4 \mathrm{~K} / \mathrm{ps}$ were examined. The obtained results suggest that the cooling rate significantly affects the average grain size and the grain size and residual stress distribution over the simulation domain. More specifically, the higher the cooling rate the lower the average grain size and the higher the average per-atom residual stress. 


\section{$\Delta$ Methodology}

The interatomic interactions were modelled using the Finnis-Sinclair (FS) potential [28], similarly to previous MD simulations [3, 23, 27]. The main advantage of the Finnis Sinclair potentials, being similar to the Embedded Atom Method (EAM) potentials, is that they consider the fact that atomic forces are dependent on the local atomic density. The FS potential can be described as follows:

$$
E_{\text {tot }}=\sum_{i=1}^{N} \sum_{j=1}^{N} V_{i j}\left(r_{i j}\right)-A \sum_{i=1}^{N} \sqrt{\rho_{i}}
$$

where $E_{t o t}$ is the total energy of the system of $N$ atoms, $V_{i j}$ the potential of the pair of atoms $i-j, r_{i j}$ the corresponding interatomic distance, $A$ the binding energy and $\rho_{i}$ the electronic charge in the vicinity of atom $i$ which is dependent on the atomic charge density $\varphi_{i j}$ :

$$
\rho_{i}=\sum_{j=1, i \neq j}^{N} \varphi_{i j}\left(r_{i j}\right)
$$

The potential energy of the pair $i-j, V_{i j}$, is given by:

$$
V_{i j}\left(r_{i j}\right)= \begin{cases}\left(r_{i j}-c\right)^{2}\left(c_{0}+c_{1} r_{i j}+c_{2} r^{2}\right) & \text { if } r_{i j} \leq c \\ 0 & \text { if } r_{i j}>c\end{cases}
$$

where $c$ is the cutoff distance and $c_{i}$ are constants employed to fit the FS potential to experimental data.

The simulation domain of this study is a cube with dimensions $25 \times 25 \times 25 \mathrm{~nm}^{3}$ containing 1 million atoms, initially located at the sites of a FCC lattice with a lattice constant equal to $4.05 \AA$. This block of atoms is initially equilibrated at $273 \mathrm{~K}$ and then isothermally annealed to a temperature of $1173 \mathrm{~K}$; this temperature value is much higher than the melting point of pure aluminium $(\approx 933 \mathrm{~K})$ while at this stage, the FCC structure has been completely replaced by liquid aluminium as shown in Figure 1. From the Common Neighbour Analysis (CNA) of Figure 1, it can be observed that during the transition from the FCC block to the equilibrated melt, a few BCC atoms are present in the simulation domain. This is in agreement with previous studies supporting that $\mathrm{Al}$ atoms pass through the $\mathrm{BCC}$ metastable phase during melting or solidification [29].

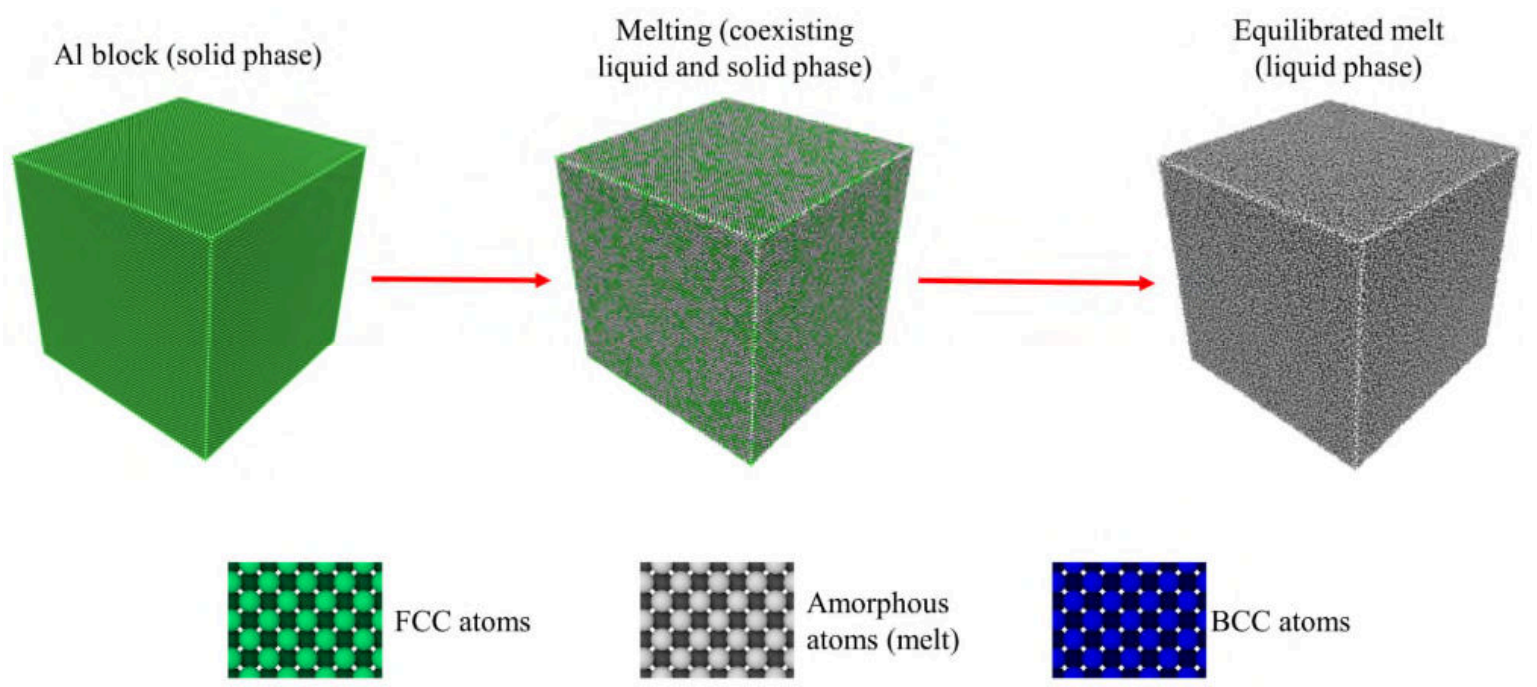

Figure 1: Melting \& Equilibration 
For the purposes of this study the equilibrated melt was then isothermally quenched back to $273 \mathrm{~K}$ under 4 different cooling rates, namely (a) $0.5 \mathrm{~K} / \mathrm{ps}$, (b) $1 \mathrm{~K} / \mathrm{ps}$, (c) $2 \mathrm{~K} / \mathrm{ps}$ and (d) $4 \mathrm{~K} / \mathrm{ps}$ and finally equilibrated for another 100,000 timesteps until the final grain structure was obtained. Periodic boundary conditions were applied to all directions. Both melting and solidification simulations were performed under the isothermal-isobaric ensemble (NPT) while the pressure and temperature of the simulation domain were controlled using a Nose-Hoover thermostat and barostat respectively. The pressure and temperature damping coefficients were 1000 and 100 timesteps, which correspond to 2 and $0.2 \mathrm{ps}$ respectively, for a timestep of $2 \mathrm{fs}$. The MD simulation parameters have been summarised in Table 1.

Table 1: MD simulation parameters

\begin{tabular}{|l|l|l|l|}
\hline Timestep (fs) & 2 & Dimensions $(\mathrm{nm})$ & $25 \times 25 \times 25$ \\
\hline Ensemble & NPT & Boundary conditions & Periodic \\
\hline Total number of atoms & $1,000,000$ & Pressure $(\mathrm{GPa})$ & 0 \\
\hline Potential & Finnis-Sinclair & Cooling rates $(\mathrm{K} / \mathrm{ps})$ & $0.5,1,2,4$ \\
\hline Cutoff distance $(\AA)$ & 6 & Equilibration time $(\mathrm{ps})$ & 200 \\
\hline
\end{tabular}

The MD simulations of this investigation were completed using the Large-scale Atomic/Molecular Massively Parallel Simulator (LAMMPS) [30] while visualisation and part of the post-processing were carried out using the Ovito Pro visualisation tool [31].

\section{Results}

As discussed in the Methodology section, the equilibrated melt was quenched under 4 different cooling rates. The potential energy evolution as a function of temperature has been plotted in Figure 2 for various cooling rates. The obtained results are similar to the ones presented in [4]. The step observed in the potential energy at $\mathrm{T} \approx 550 \mathrm{~K}$ denotes the transition from solid to liquid phase. The aforementioned step becomes smoother and this transition occurs over a larger temperature range as the cooling rate increases; this is due to rapid cooling. Finally, it can be observed that the absolute value of the final peratom potential energy $(\mathrm{T}=273 \mathrm{~K})$ becomes higher as the cooling rate decreases; this is due to the fact the slower quenching leads to fewer grains and consequently reduced grain boundary area. In other words, slower cooling rates promote the formation of the FCC phase which is energetically favourable [26].

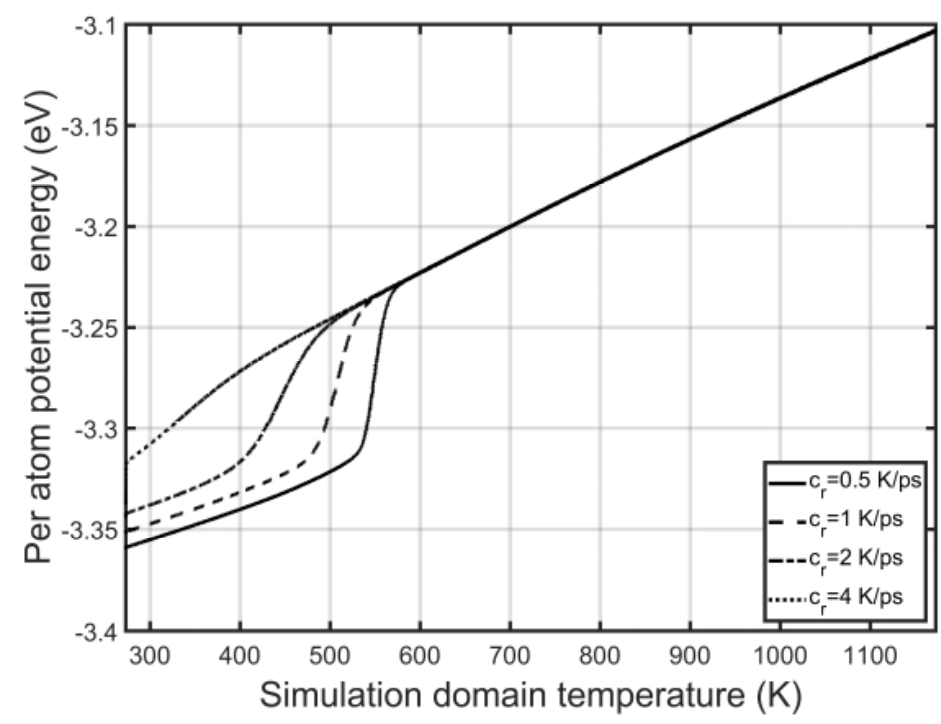

Figure 2: Per-atom potential energy vs. temperature for various cooling rates 
Nucleation and grain size evolution have been visualised in Figure 3 at various stages of solidification (a-c) and at the end of the equilibration process (d) for a cooling rate $c_{r}$ equal to $1 \mathrm{~K} / \mathrm{ps}$. The grain size was measured by assuming that all grains are spherical and the grain diameter $d_{g}$ was calculated according to:

$$
\frac{4}{3} \pi\left(\frac{d_{g}}{2}\right)^{3}=N V_{a} \Leftrightarrow d_{g}=2 \sqrt[3]{\frac{3 N V_{a}}{4 \pi}}
$$

where $N$ is the number of atoms contained in a single grain and $V_{a}$ the per-atom volume, calculated using the Voronoi analysis module of Ovito [32]. As it can be observed from Figure 3 some of the embryos formed initially (Figure 3(a)) manage to grow in size and become grains which later on further grow in size simultaneously until the final microstructure has been obtained.

(a)

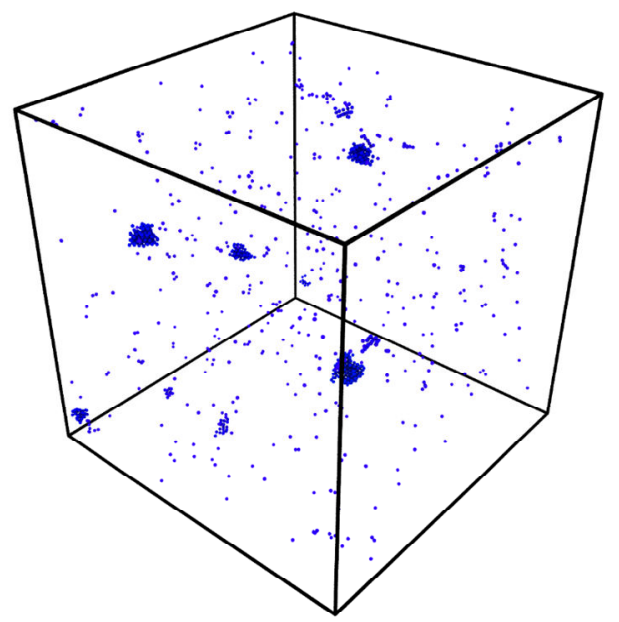

(c)

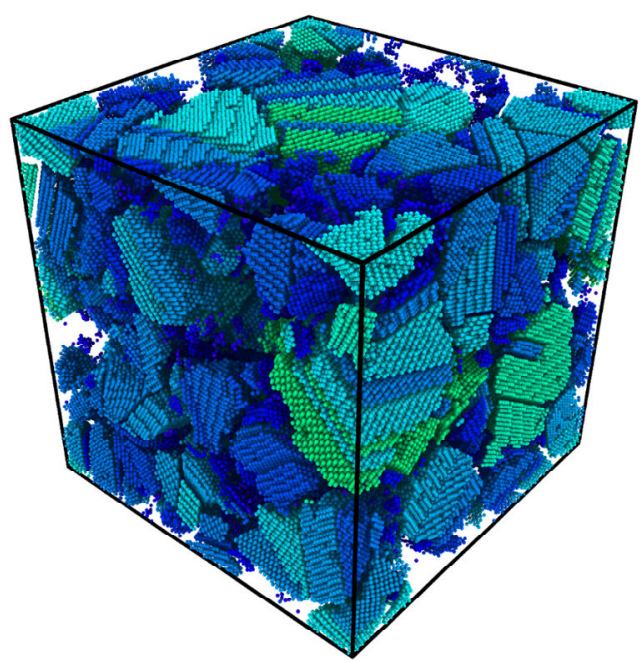

(b)

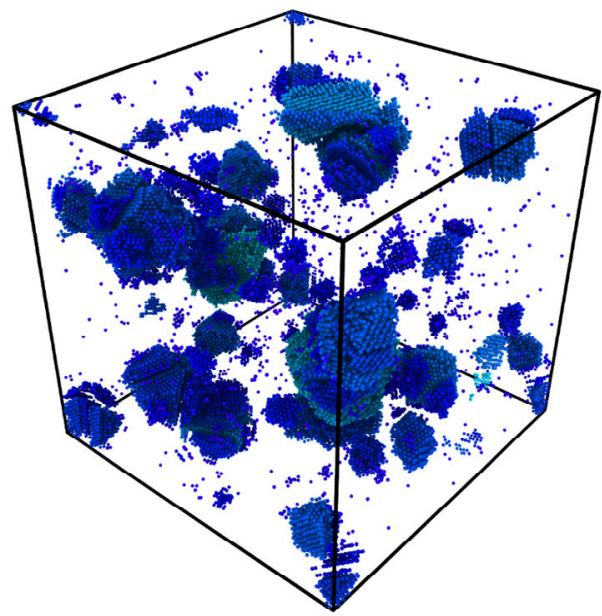

(d)

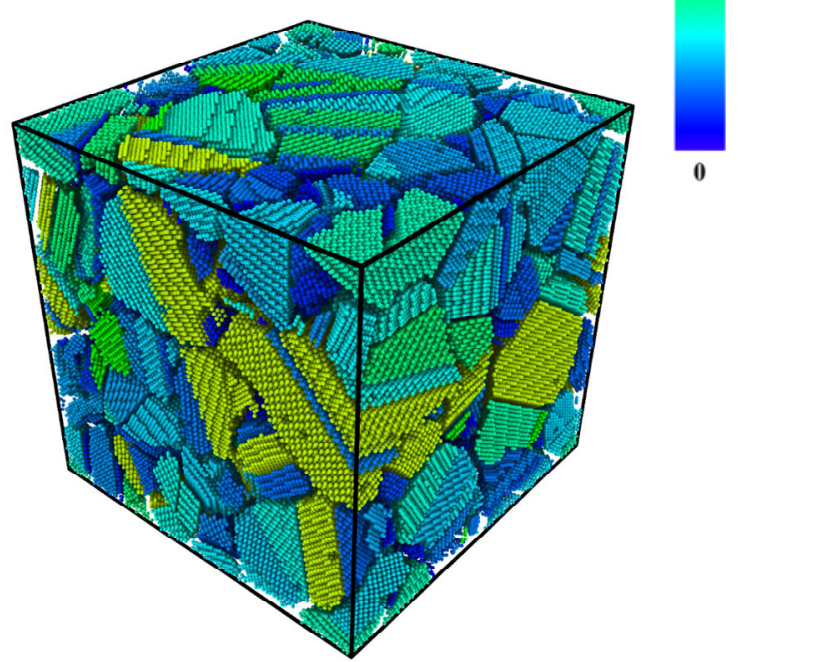

Figure 3: Nucleation and grain size evolution at $t=(a) 612 \mathrm{ps,}$, (b) $648 \mathrm{ps}$, (c) $684 \mathrm{ps}$ and (d) $1120 \mathrm{ps}$ (end of equilibration) for $c_{r}=1 \mathrm{~K} / \mathrm{ps}$

The final microstructures obtained at the end of the equilibration stage for the various cooling rates examined have been represented in Figure 4. It is evident that the higher the cooling rate the smaller and the more the grains of the equilibrated structure. 
(a)

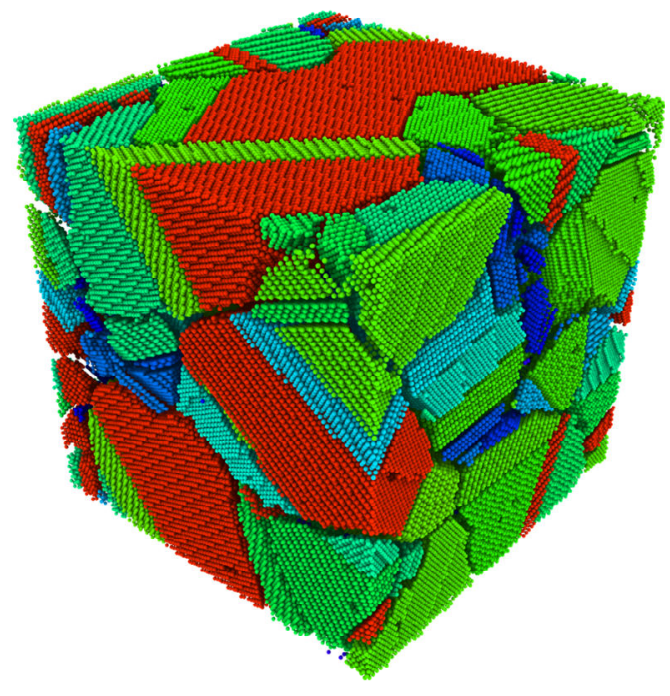

(c)

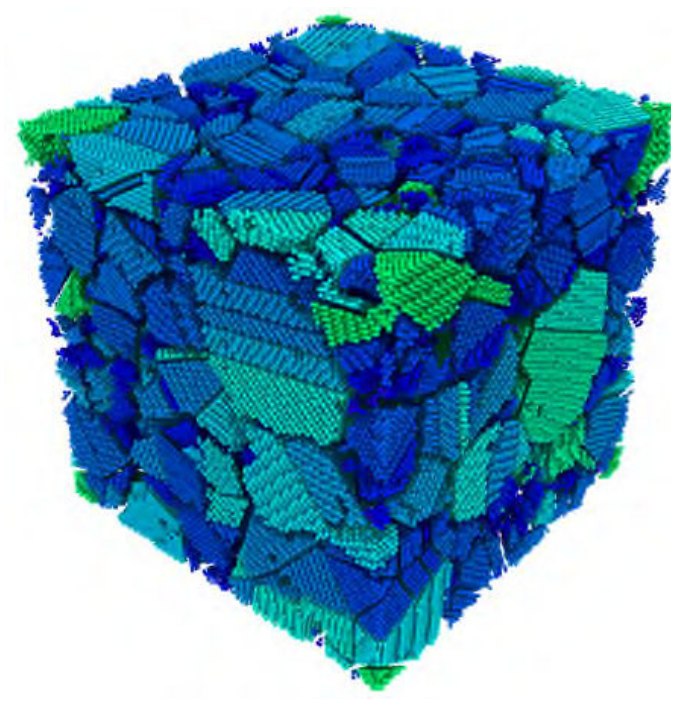

(b)

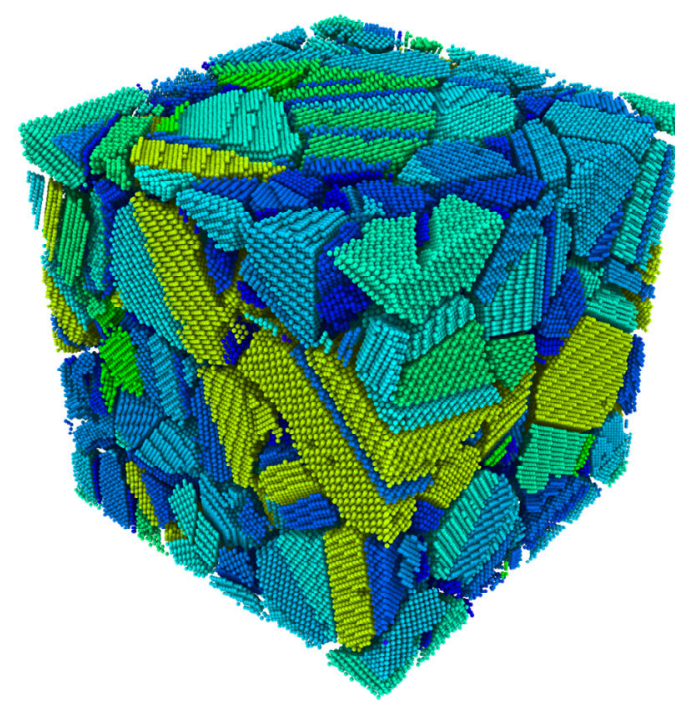

(d)

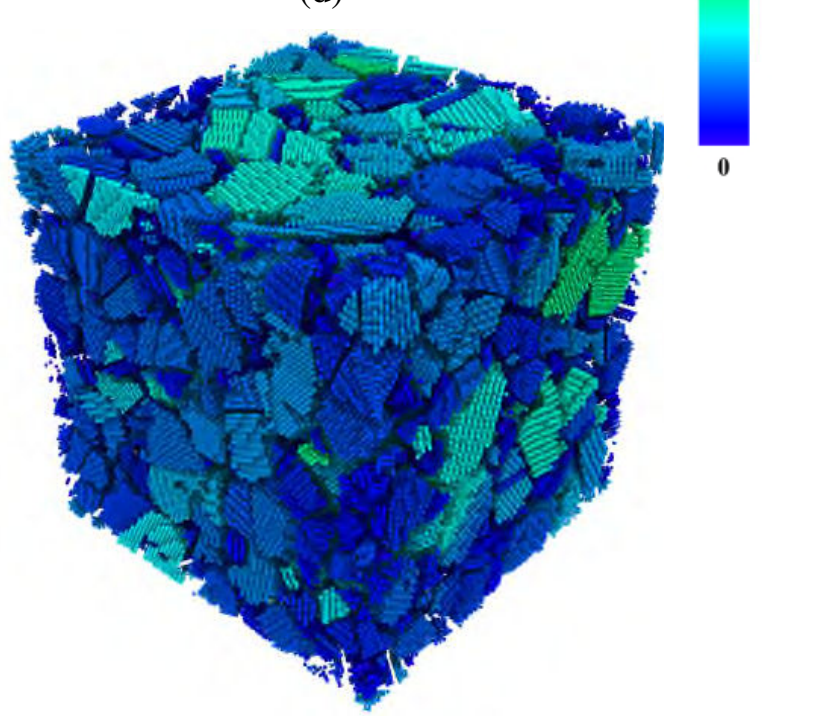

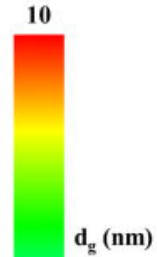

$d_{g}(n m)$

Figure 4: Final grain structure for $c_{r}=($ a) $0.5 \mathrm{~K} / \mathrm{ps}$, (b) $1 \mathrm{~K} / \mathrm{ps}$, (c) $2 \mathrm{~K} / \mathrm{ps}$ and (d) $4 \mathrm{~K} / \mathrm{ps}$

Figures 3 and 4 are just illustrations of the nucleation and equilibration stages while they don't provide any quantitative information on the dependence of the average grain size or grain size distribution on the cooling rate. For the purposes of this analysis, the average grain diameter $d_{g, a v e}$ has been plotted as a function of the cooling rate in Figure 5; the $d_{g, a v e}$ values of this graph correspond to the average diameter of the grains as measured at the last timestep of the equilibration stage. It can be observed that the average grain size drops parabolically with the cooling rate. A cooling rate of $0.5 \mathrm{~K} / \mathrm{ps}$ leads to an average grain diameter $d_{g, a v e}$ about equal to $4.2 \mathrm{~nm}$ while this value reduces to $2 \mathrm{~nm}$ for a cooling rate of $4 \mathrm{~K} / \mathrm{ps}$. It has to be noted that the average grain diameter appears to be quite small when compared against Figure 4; this is due to the fact that there are numerous tiny grains lying in the interstices of larger ones which further reduce the $d_{g, a v e}$ value. 


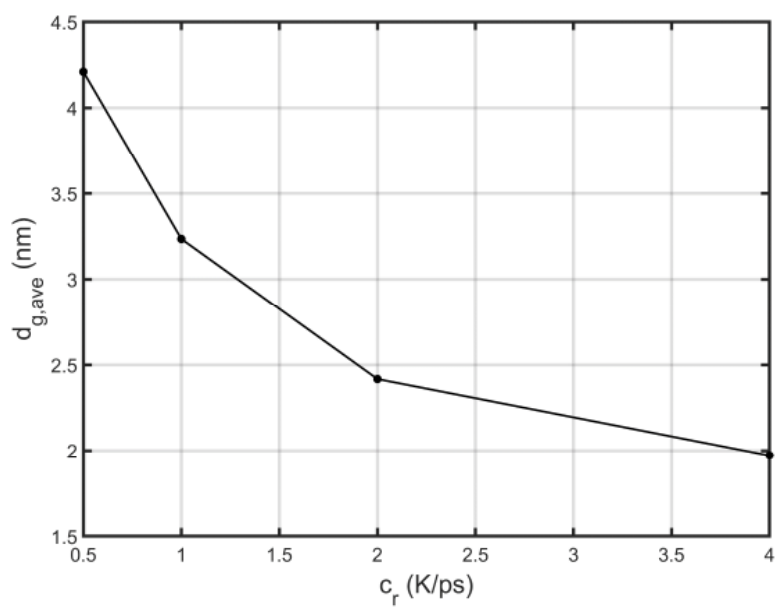

Figure 5: Average grain diameter $d_{g, a v e}$ (measured at the end of equilibration stage) as a function of the cooling rate $c_{r}$

In addition to the analysis above, the grain size distribution has been evaluated for the various cooling rates under investigation and illustrated in Figure 6. Grains have been categorised based on their grain diameter and the population of each category has been estimated. It can be observed that the lower the cooling rate the wider the grain diameter distribution. For a cooling rate of $0.5 \mathrm{~K} / \mathrm{ps}$ the maximum grain diameter observed was $19.97 \mathrm{~nm}$ while for a cooling rate of $4 \mathrm{~K} / \mathrm{ps}$ the maximum grain diameter was less than half of this value $(8.95 \mathrm{~nm})$. Finally, the number of small grains increases sharply with the cooling rate; just 50 grains with a diameter of $0.69 \mathrm{~nm}$ were observed for $\mathrm{c}_{\mathrm{r}}=0.5 \mathrm{~K} / \mathrm{ps}$ while this value climbed to 900 for $\mathrm{c}_{\mathrm{r}}=4 \mathrm{~K} / \mathrm{ps}$.

(a)

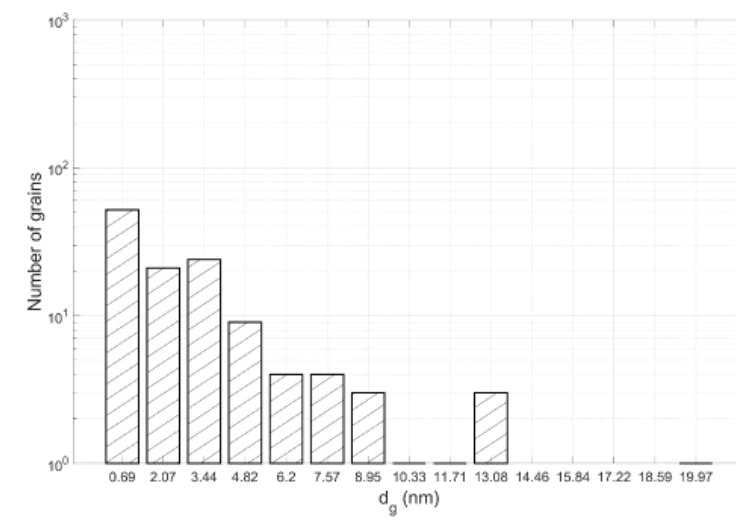

(c)

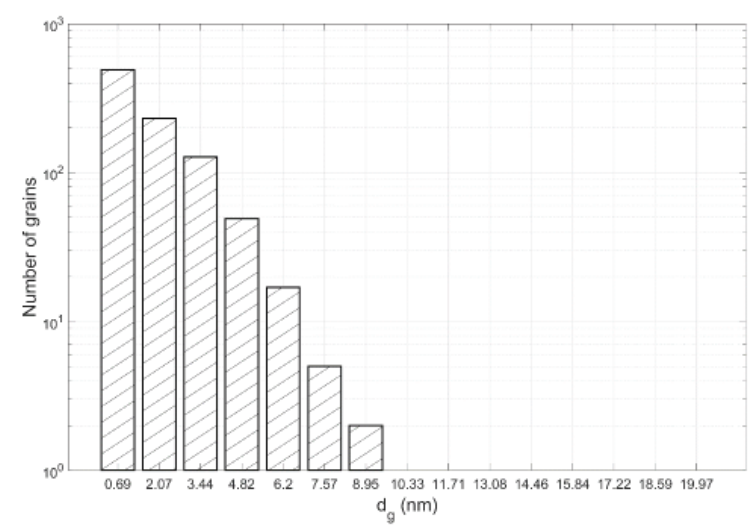

(b)

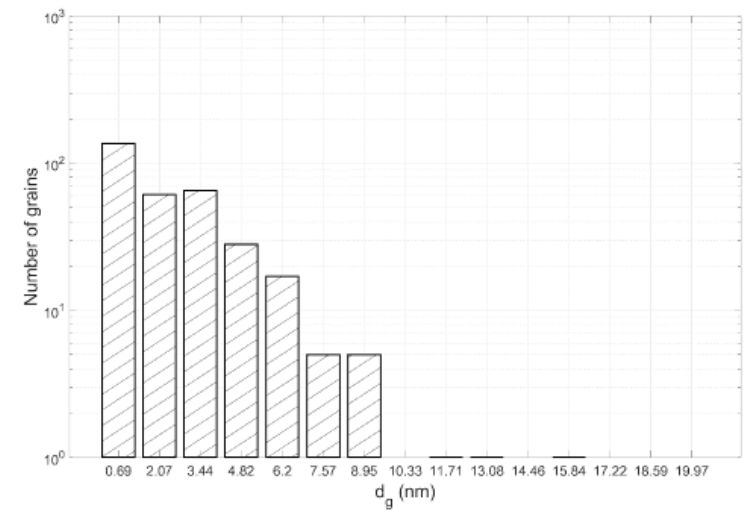

(d)

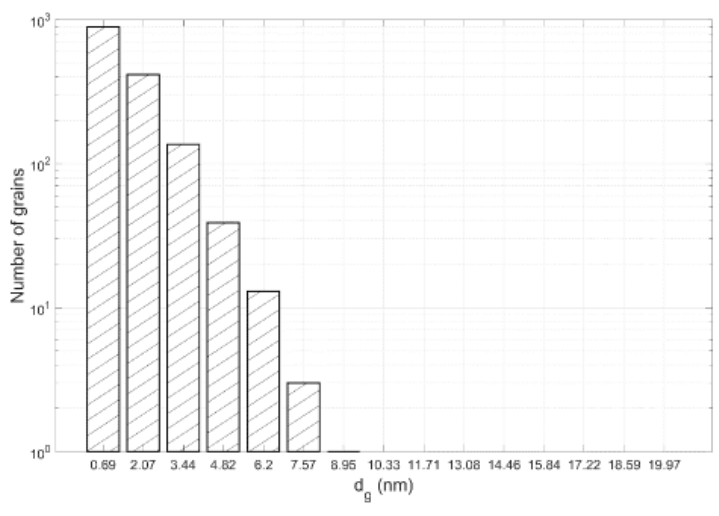

Figure 6: Grain size distribution for $c_{r}=(a) 0.5 \mathrm{~K} / \mathrm{ps}$, (b) $1 \mathrm{~K} / \mathrm{ps}$, (c) $2 \mathrm{~K} / \mathrm{ps}$ and (d) $4 \mathrm{~K} / \mathrm{ps}$ 
The last part of this investigation is focused on the distribution and estimation of residual stresses over the simulation domain. Residual stresses were estimated using the equivalent Von Misses (VM) stress as described below:

$$
\sigma_{V M}=\sqrt{\frac{\left(\sigma_{x x}-\sigma_{y y}\right)^{2}+\left(\sigma_{y y}-\sigma_{z z}\right)^{2}+\left(\sigma_{z z}-\sigma_{x x}\right)^{2}+6\left(\sigma_{x y}{ }^{2}+\sigma_{y z}{ }^{2}+\sigma_{z x}{ }^{2}\right)}{2}}
$$

where $\sigma_{x x}, \sigma_{y y}, \sigma_{z z}$ the principal and $\sigma_{x y}, \sigma_{y z}$ and $\sigma_{z x}$ the shear stresses respectively. The per-atom VM stress components were averaged over time widows in order to flatten the noise.

(a)

Common Neighbour Analysis

(b)

(c)

(d)
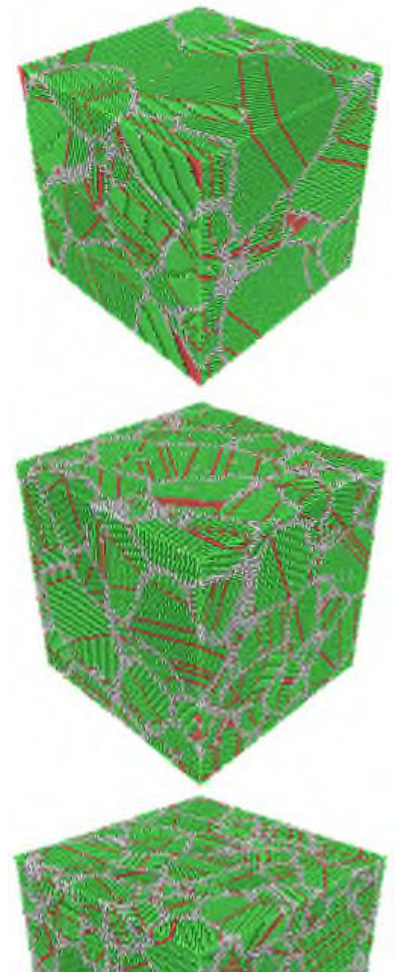

)
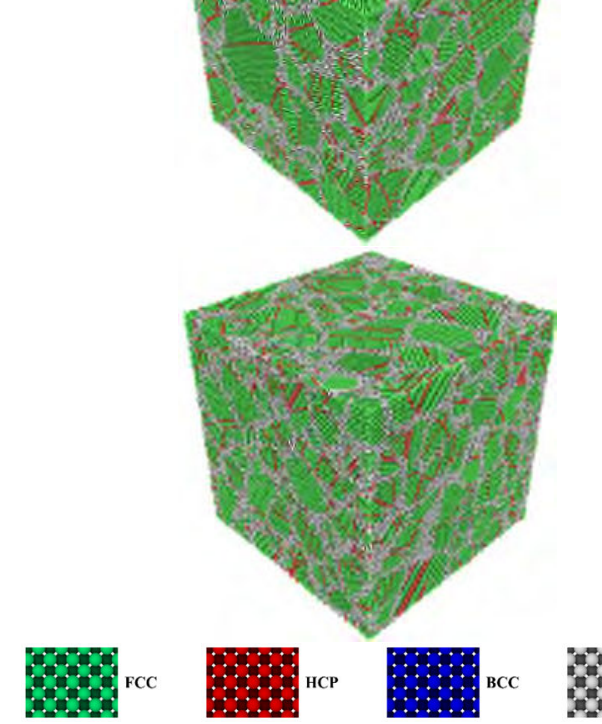

Figure 7: Common Neighbour Analysis (CNA) and VM stress distribution at the final timestep of the equilibration stage

The distribution of VM stresses over the simulation domain and the corresponding CNA analysis snapshots has been illustrated in Figure 7. By comparing the results it can be concluded that higher residual stresses are localised at the grain boundaries; this result is in agreement with previous 
experimental investigations [33] and according to the authors' best knowledge this is the first time that this has been captured via means of MD simulations. The residual stresses appear to be equal to zero at the area of the grains, where the FCC phase is prevalent. Additionally, it can be observed that residual stresses are high in the vicinity of lattice dislocations which are denoted by the Hexagonal Close Packed (HCP) atoms; this result is also in agreement with [34]. However, the stresses at the vicinity of lattice dislocations are significantly lower than the ones at the grain boundaries.

In order to further investigate the evolution of the residual stresses we have calculated the average peratom $\mathrm{VM}$ residual stresses using the following ensemble average $\left(\sigma_{V M, \text { ave }}\right)$ :

$$
\sigma_{V M, a v e}=\frac{1}{N} \sum_{i=1}^{N} \sigma_{V M, i}
$$

where $N$ is total number of atoms. The average VM stress has been plotted as a function of time in Figure 8 over the solidification and equilibration stages for various cooling rates. It can be observed that when quenching commences $\sigma_{V M, \text { ave }}$ obtains a temporarily constant value which appears to be proportional to the cooling rate of each simulation. This can be explained if someone considers an analogue of the thermal stresses being proportional to the cooling rate. Later on, $\sigma_{V M \text {, ave }}$ starts increasing sharply and reaches a constant value after experiencing some fluctuations. As expected, the final value of the residual stresses, expressed in terms of VM stress in this study, is higher for higher values of the cooling rate due to the increased area of grain boundaries and lattice dislocations.

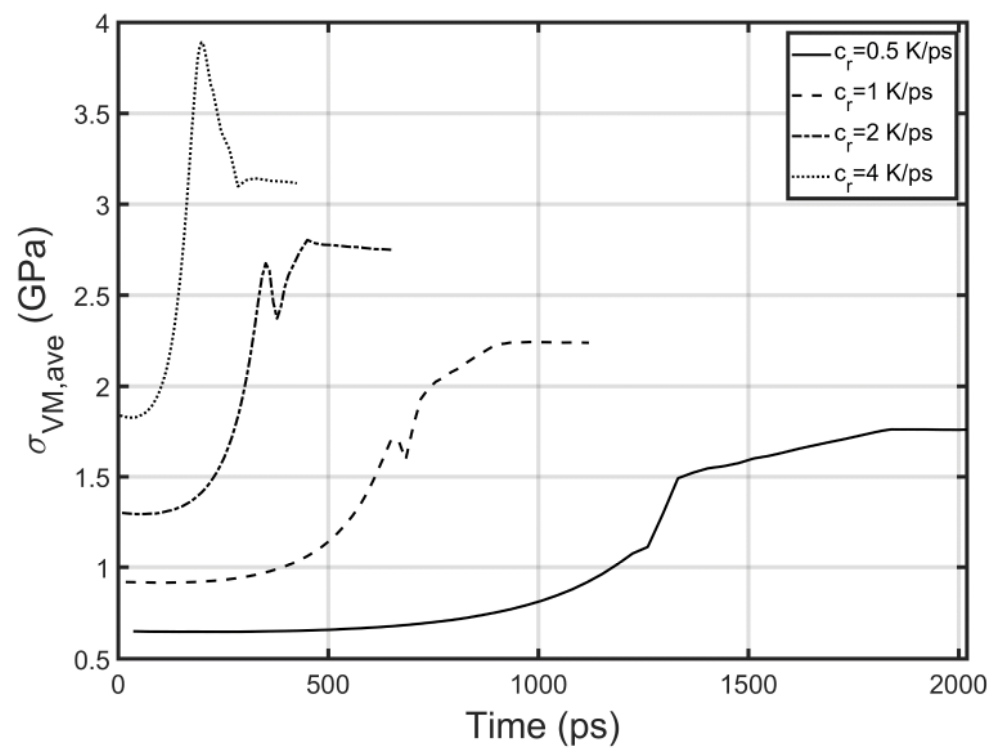

Figure 8: Average $\sigma_{V M}$ vs. time

In order to understand the reason behind the aforementioned fluctuations in the residual stresses the population of grains as a function of time has been plotted in Figure 9. By comparing Figure 8 and Figure 9 it can be seen that residual stresses increase sharply right at the time when the grain population follows the same trend. However, as advocated by previous studies, solidification is an exothermic reaction followed by the release of the latent heat [26]; this leads to localised elevated temperature regions and some of the grains, merge with the neighbouring ones. This is also known as the coarsening stage [4]. This form of local heat treatment is the reason for the rapid reduction of the grain population after it reaches its peak. For the exact same reason the residual stresses drop at the same time interval until they reach a constant value. This phenomenon is more pronounced for higher cooling rates as the latent heat is released over a much shorter time interval. This is the reason why this intermediate peak in residual stresses cannot be observed for the lowest value of the cooling rate $c_{r}=0.5 \mathrm{~K} / \mathrm{ps}$ (see Figure 8). Following their peak, residual stresses follow their increasing trend for a short period of time before 
obtaining a constant value as the absolute value of the potential energy of the system further increases during equilibration.

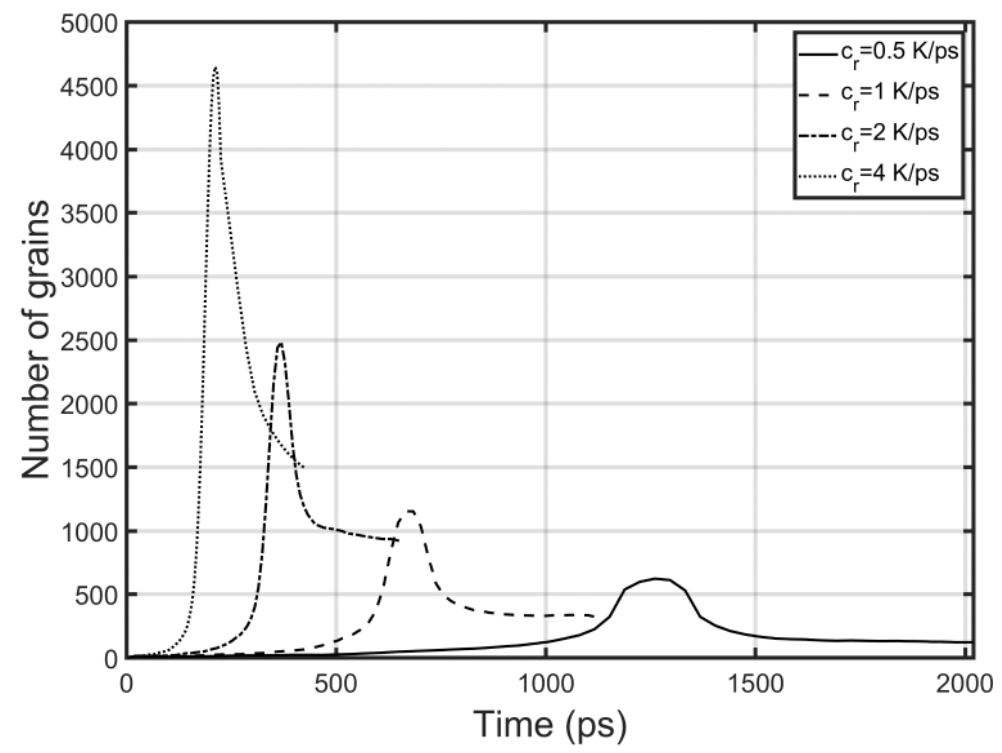

Figure 9: Number of grains vs. time

\section{Conclusions}

In this investigation large scale 3-dimensional Molecular Dynamics simulations have been performed to investigate rapid solidification of pure Aluminium under various cooling rates. Special emphasis was placed on the microstructure development during the solidification and equilibration stages as well as the stress evolution and distribution over the simulation domain. The main conclusions drawn are summarised below:

- The grain distribution can be effectively controlled by tuning the cooling rate. Higher cooling rates lead to a large number of small grains while low ones to a small number of larger grains.

- High residual stresses are localised at the vicinity of grain boundaries and lattice dislocations. This is the first time that this phenomenon has been captured via MD simulations according to the authors' best knowledge.

- High cooling rates lead to increased residual stresses. This is due to the increased grain boundary area and the additional lattice dislocations induced during quenching.

- The grain coarsening phenomenon has been captured via MD simulations.

- Solidification simulations should allow for additional time after quenching for the simulation domain to equilibrate. This additional time is required in order to observe the grain coarsening phenomenon and have a more accurate estimation of the final number of grains and their distribution in the solidified microstructure.

\section{$\ulcorner$ Acknowledgements}

This work has been funded by the UK EPSRC project "Energy Resilient Manufacturing 2: Small is Beautiful Phase 2 (SIB2)" (EP/P012272/1) 


\section{References}

1. Hall EO (1951) The Deformation and Ageing of Mild Steel: III Discussion of Results. Proc Phys Soc Sect B 64:747-753. https://doi.org/10.1088/0370-1301/64/9/303

2. Petch NJ (1953) The cleavage strength of polycrystals. J Iron Steel Inst 174:25-28

3. Papanikolaou, Salonitis, Jolly, Frank (2019) Large-Scale Molecular Dynamics Simulations of Homogeneous Nucleation of Pure Aluminium. Metals (Basel) 9:1217. https://doi.org/10.3390/met9111217

4. Hou ZY, Dong KJ, Tian ZA, et al (2016) Cooling rate dependence of solidification for liquid aluminium: a large-scale molecular dynamics simulation study. Phys Chem Chem Phys 18:17461-17469. https://doi.org/10.1039/C6CP02172G

5. Dong C, Wu H bin, Wang X tao (2019) Effect of cooling rate on microstructure, hardness, and residual stress of $0.28 \mathrm{C}-0.22 \mathrm{Ti}$ wear-resistant steel. J Iron Steel Res Int 26:866-874. https://doi.org/10.1007/s42243-019-00283-1

6. Dolan GP, Robinson JS (2004) Residual stress reduction in 7175-T73, 6061-T6 and 2017A-T4 aluminium alloys using quench factor analysis. J Mater Process Technol 153-154:346-351. https://doi.org/10.1016/j.jmatprotec.2004.04.065

7. Sear RP (2006) Heterogeneous and homogeneous nucleation compared: Rapid nucleation on microscopic impurities. J Phys Chem B 110:4985-4989. https://doi.org/10.1021/jp056377e

8. Oxtoby DW (1992) Homogeneous nucleation: Theory and experiment. J Phys Condens Matter 4:7627-7650. https://doi.org/10.1088/0953-8984/4/38/001

9. Iqbal N, van Dijk NH, Offerman SE, et al (2005) Real-time observation of grain nucleation and growth during solidification of aluminium alloys. Acta Mater 53:2875-2880. https://doi.org/10.1016/J.ACTAMAT.2005.02.045

10. Erdemir D, Lee AY, Myerson AS (2009) Nucleation of crystals from solution: Classical and two-step models. Acc Chem Res 42:621-629. https://doi.org/10.1021/ar800217x

11. Vekilov PG (2010) The two-step mechanism of nucleation of crystals in solution. Nanoscale 2:2346-2357. https://doi.org/10.1039/c0nr00628a

12. Gavezzotti A (1999) Molecular aggregation of acetic acid in a carbon tetrachloride solution: A molecular dynamics study with a view to crystal nucleation. Chem - A Eur J 5:567-576. https://doi.org/10.1002/(SICI)1521-3765(19990201)5:2<567::AID-CHEM567>3.0.CO;2-6

13. Talanquer V, Oxtoby DW (1998) Crystal nucleation in the presence of a metastable critical point. J Chem Phys 109:223-227. https://doi.org/10.1063/1.476554

14. Knezic D, Zaccaro J, Myerson AS (2004) Nucleation induction time in levitated droplets. J Phys Chem B 108:10672-10677. https://doi.org/10.1021/jp049586s

15. Del Vitto A, Pacchioni G, Delbecq F, Sautet P (2005) Au atoms and dimers on the $\mathrm{MgO}(100)$ surface: a DFT study of nucleation at defects. J Phys Chem B 109:8040-8048

16. Nie Y, Liang C, Cha P-R, et al (2017) A kinetic Monte Carlo simulation method of van der Waals epitaxy for atomistic nucleation-growth processes of transition metal dichalcogenides. Sci Rep 7:2977

17. Qin RS, Bhadeshia HKDH (2009) Phase-field model study of the effect of interface anisotropy on the crystal morphological evolution of cubic metals. Acta Mater 57:2210-2216. https://doi.org/10.1016/J.ACTAMAT.2009.01.024

18. Tian Z-A, Liu R-S, Zheng C-X, et al (2008) Formation and Evolution of Metastable bec Phase during Solidification of Liquid Ag: A Molecular Dynamics Simulation Study. J Phys Chem A 112:12326-12336. https://doi.org/10.1021/jp804836b 
19. Tian Z-A, Liu R-S, Liu H-R, et al (2008) Molecular dynamics simulation for cooling rate dependence of solidification microstructures of silver. J Non Cryst Solids 354:3705-3712. https://doi.org/10.1016/J.JNONCRYSOL.2008.04.006

20. Sutton AP, Chen J (1990) Long-range finnis-sinclair potentials. Philos Mag Lett 61:139-146. https://doi.org/10.1080/09500839008206493

21. Streitz FH, Glosli JN, Patel M V (2006) Beyond finite-size scaling in solidification simulations. Phys Rev Lett 96:225701

22. Mahata A, Zaeem MA, Baskes MI (2018) Understanding homogeneous nucleation in solidification of aluminum by molecular dynamics simulations. Model Simul Mater Sci Eng 26:025007. https://doi.org/10.1088/1361-651X/aa9f36

23. Shibuta Y, Sakane S, Takaki T, Ohno M (2016) Submicrometer-scale molecular dynamics simulation of nucleation and solidification from undercooled melt: Linkage between empirical interpretation and atomistic nature. Acta Mater 105:328-337. https://doi.org/10.1016/J.ACTAMAT.2015.12.033

24. Matsui T, Ogawa T, Adachi Y (2019) Relationship between three-dimensional microstructure and Avrami exponent for recrystallization in pure iron. Results Mater 1:100002. https://doi.org/10.1016/j.rinma.2019.100002

25. Shibuta Y, Sakane S, Miyoshi E, et al (2017) Heterogeneity in homogeneous nucleation from billion-atom molecular dynamics simulation of solidification of pure metal. Nat Commun 8:10. https://doi.org/10.1038/s41467-017-00017-5

26. Papanikolaou M, Salonitis K, Jolly M, Frank M (2019) Large-Scale Molecular Dynamics Simulations of Homogeneous Nucleation of Pure Aluminium. Metals (Basel) 9:1217

27. Papanikolaou M, Salonitis K, Jolly M (2020) Molecular Dynamics Simulations of the Solidification of Pure Aluminium. In: Minerals, Metals and Materials Series. Springer, pp 158167

28. Finnis MW, Sinclair JE (1984) A simple empirical N-body potential for transition metals. Philos Mag A 50:45-55

29. Rein ten Wolde P, Ruiz-Montero MJ, Frenkel D (1996) Numerical calculation of the rate of crystal nucleation in a Lennard-Jones system at moderate undercooling. J Chem Phys 104:99329947. https://doi.org/10.1063/1.471721

30. LAMMPS Molecular Dynamics Simulator. https://lammps.sandia.gov/. Accessed 12 Nov 2019

31. Stukowski A (2015) Ovito Open Visualization Tool

32. Stukowski A (2012) Structure identification methods for atomistic simulations of crystalline materials. Model Simul Mater Sci Eng 20:45021

33. Vedula VR, Glass SJ, Saylor DM, et al (2001) Residual-Stress Predictions in Polycrystalline Alumina. J Am Ceram Soc 84:2947-2954. https://doi.org/10.1111/j.1151-2916.2001.tb01119.x

34. Papanikolaou M, Hernandez FR, Salonitis K (2020) Investigation of the Subsurface Temperature Effects on Nanocutting Processes via Molecular Dynamics Simulations. Metals (Basel) 10:1220. https://doi.org/10.3390/met10091220 
$2021-02-24$

\title{
Molecular dynamics simulations of the evolution of residual stresses during rapid solidification of aluminium
}

\author{
Papanikolaou, Michail
}

Springer

Papanikolaou M, Salonitis K, Jolly M. (2021) Molecular dynamics simulations of the evolution of residual stresses during rapid solidification of aluminium. In: 2021 TMS Annual Meeting \& Exhibition: TMS2021,15-18 March 2021, Virtual Event https://doi.org/10.1007/978-3-030-65396-5_122

Downloaded from Cranfield Library Services E-Repository 